\title{
The effects of metallicity on the Galactic disk population of white dwarfs
}

\author{
Ruxandra Cojocaru $^{1,2}$, Santiago Torres ${ }^{1,2}$, Jordi Isern ${ }^{3,2}$, and Enrique García-Berro ${ }^{1,2}$ \\ ${ }^{1}$ Departament de Física Aplicada, Universitat Politècnica de Catalunya, c/Esteve Terrades 5, 08860 Castelldefels, Spain \\ e-mail: enrique.garcia-berro@upc.edu \\ 2 Institute for Space Studies of Catalonia, c/Gran Capità 2-4, Edif. Nexus 104, 08034 Barcelona, Spain \\ 3 Institut de Ciències de l'Espai (CSIC), Campus UAB, Facultat de Ciències, Torre C-5 08193 Bellaterra, Spain
}

Received 10 January 2014 / Accepted 28 April 2014

\section{ABSTRACT}

\begin{abstract}
Context. It has been known for a long time that stellar metallicity plays a significant role in the determination of the ages of the different Galactic stellar populations, when main sequence evolutionary tracks are employed.

Aims. Here, we analyze the role that metallicity plays on the white dwarf luminosity function of the Galactic disk, which is often used to determine its age.

Methods. We employ a Monte Carlo population synthesis code that accounts for the properties of the population of Galactic disk white dwarfs. Our code incorporates the most up-to-date evolutionary cooling sequences for white dwarfs with hydrogen-rich and hydrogen-deficient atmospheres for both carbon-oxygen and oxygen-neon cores. We use two different models to assess the evolution of the metallicity: the first, where the adopted metallicity is constant with time but with a moderate dispersion, and the second where the metallicity increases with time.

Results. We found that our theoretical results are in a very satisfactory agreement with the observational luminosity functions obtained from the Sloan Digital Sky Survey (SDSS) and from the SuperCOSMOS Sky Survey (SSS). These results are independent of the adopted age-metallicity law. In particular, we found that the age-metallicity law has no noticeable impact in shaping the bright branch of the white dwarf luminosity function and that the position of its cut-off is almost insensitive to the adopted age-metallicity relationship.

Conclusions. Because the shape of the bright branch of the white dwarf luminosity function is insensitive to the age-metallicity law, it can be safely employed to test the theoretical evolutionary sequences, while its location provides a robust indicator of the age of the Galactic disk due to the limited sensitivity of the position of the drop-off to the distribution of metallicities.
\end{abstract}

Key words. white dwarfs - stars: luminosity function, mass function - Galaxy: evolution - Galaxy: abundances

\section{Introduction}

The evolutionary properties of white dwarfs have been the subject of intensive studies over the past three decades. This is partially because these fossil stars convey important information about the ages, formation, and evolution of a wide range of Galactic stellar populations. White dwarfs are the evolutionary endpoint of most stars in our Galaxy. Specifically, white dwarfs are the direct descendants of main-sequence stars with masses ranging from $\sim 0.8 M_{\odot}$ to $\sim 10 M_{\odot}$. Given the shape of the initial mass function, a straightforward calculation shows that over $98 \%$ of the stars in our Galaxy that have main-sequence evolutionary lifetimes shorter than its age ( $10 \mathrm{Gyr})$ become white dwarfs. In addition to being so numerous, white dwarfs have other interesting properties. Specifically, the two most important are their structural simplicity and their well-known evolutionary properties - see, e.g., Althaus et al. (2010a). This result and the fact that the evolution of white dwarfs is a simple and well-studied cooling process, in which gravothermal energy is slowly radiated away, have allowed white dwarfs to be used as independent cosmic clocks. In particular, white dwarfs have been employed to estimate the ages of a variety of Galactic stellar populations, such as the Galactic disk (Winget et al. 1987; García-Berro et al. 1988) and halo (Mochkovitch et al. 1990; Isern et al. 1998) or the system of Galactic globular (Kalirai et al. 2001; Hansen et al. 2002, 2013) and open clusters (see, e.g., García-Berro et al. 2010; or Jeffery et al. 2011 for recent studies). Furthermore, the ensemble properties of the population of Galactic disk white dwarfs have also been used to test theoretical scenarios and theories that cannot be probed yet in terrestrial laboratories. These include, for instance, testing alternative theories of gravitation, which result in a hypothetical variation of the gravitational constant (Garcia-Berro et al. 1995, 2011), setting constraints on the mass of weakly interacting particles like axions (Isern et al. 2008), or constraining the properties of the so-called dark forces (Dreiner et al. 2013). Additionally, the population of white dwarfs has also been used to derive interesting constraints on the local star-formation history (Noh \& Scalo 1990; Diaz-Pinto et al. 1994; Rowell 2013). Finally, there is a plethora of studies dedicated to extensively analyze the contribution of white dwarfs to the baryonic dark matter content of our Galaxy (see, for instance, Kawaler 1996; Torres et al. 2002; Pauli et al. 2003; or García-Berro et al. 2004, and references therein), which is a topic of the maximum interest.

To obtain useful information from the different populations of white dwarfs, three conditions must be fulfilled. First, accurate observational data are needed. With the advent of large-scale automatic surveys, like the Sloan Digital Sky survey (Gunn et al. 1998a) or the SuperCOSMOS Sky Survey (Rowell \& Hambly 2011), to cite just two representative examples, the sample of white dwarfs with reliable and accurate measurements of their astronomical properties has largely increased, thus allowing for 
detailed comparisons with theoretical models. The second important ingredient to obtain useful information from the observed populations of Galactic white dwarfs is a set of accurate evolutionary cooling sequences. The degree of accuracy and sophistication of the available cooling sequences has also been considerably improved during the last decade, and modern evolutionary sequences include a detailed treatment of the atmospheres (Rohrmann et al. 2012), as well as an accurate treatment of all the sinks and sources of energy in the deep interior of white dwarfs (Isern et al. 1997, 2000). Among other interesting physical phenomena, we mention neutrino emission, ${ }^{22} \mathrm{Ne}$ diffusion in the liquid phase (Isern et al. 1991; Althaus et al. 2010b), and phase separation of the carbon-oxygen binary mixture upon crystallization (Segretain et al. 1994). Moreover, it is worth emphasizing that the agreement between the cooling tracks computed using different stellar evolutionary codes is nowadays excellent, and even better than that found between different mainsequence evolutionary sequences (see Salaris et al. 2013, for a detailed discussion of this issue). Finally, a tool to model the ensemble properties of these populations is also needed. For this, the best choice is a Monte Carlo simulator, a technique which is frequently used to model the different Galactic stellar populations. Monte Carlo techniques have been frequently used to model the population of disk white dwarfs, compensating the poor statistics of the old observational samples at times (García-Berro et al. 1999, 2004) and allowing one to study the effects of biases and of the sample selection procedure (Geijo et al. 2006; Torres et al. 2007).

Cosmic age determinations are usually obtained by employing models and evolutionary tracks for stars with solar metallicity, which is the median value for the metallicity distribution function (MDF) of the Galactic disk. However, the entire span of this distribution function is many times neglected. Over the years, photometric studies of main sequence stars have resulted in the release of large catalogs (Twarog 1980; Olsen 1983; Strömgren 1987; Nordström et al. 2004; Haywood 2008). In most occasions these catalogs contain one parameter for metallicity and no detailed element abundances. Perhaps, one of the most important results of these surveys is that high-metallicity stars are present throughout the entire mass range and that the MDF depends on the stellar age. Even if the peak value for the metallicity remains approximately at solar values, young stars have a much narrower distribution than old stars, which suggests that a correct sampling should be denser for younger stars (Casagrande et al. 2011). The rather symmetric shape of the MDF is explained through the natural process of star migration (Sellwood \& Binney 2002), in which high-metallicity stars migrate from the inner disk and low-metallicity stars do so from the outer disk. Additionally, it is believed that old stars probably play a more significant role in this situation, given that they clearly contribute to the lower metallicity wing of the MDF (Casagrande et al. 2011). This may have important consequences. On the one hand, recent studies have shown that high values of the metallicity imply slower evolutionary rates for moderately cool white dwarfs due to the sedimentation of ${ }^{22} \mathrm{Ne}$ (Isern et al. 1991; García-Berro et al. 2010; Althaus et al. 2010b). On the other hand, metallicity modifies the lifetime of white dwarf progenitors, and there is not yet a consensus of whether the initial final mass relationship is modified (see Isern et al. 2005, for a preliminary discussion). Obviously, these effects may affect the age determination of the Galactic disk obtained using the faint downturn of the white dwarf luminosity function. The ultimate goal of this work is to assess how the evolution of the metallicity of the Galactic disk affects the shape of the white dwarf luminosity function.

To accomplish this aim, we employ two metallicity laws. The first of them is a metallicity law with a median corresponding to the solar value and a dispersion around it (Casagrande et al. 2011), whereas the second is the classical age-metallicity relationship of Twarog (1980). The paper is organized as follows. In Sect. 2, we explain the most basic features of our population synthesis code, while we describe the two most recent and reliable observational samples with which we compare our simulated data in Sect. 3. We then discuss the selection criteria employed to cull a representative sample of white dwarfs from these surveys. Section 4 is devoted to explaining in depth the results of our Monte Carlo simulations and to thoroughly compare our population synthesis results with the observational data. Finally, we review our most relevant findings, discuss their significance, and summarize our conclusions in Sect. 5.

\section{The population synthesis code}

A full description of all the relevant ingredients employed in our Monte Carlo simulator can be found in our previous works (García-Berro et al. 1999, 2004; Torres et al. 2002). However, for the sake of completeness, we summarize our main inputs here. The simulations described in this paper were done using a random number generator algorithm (James 1990), which provides a uniform probability density within the interval $(0,1)$ and ensures a repetition period of $\gtrsim 10^{18}$, which is virtually infinite for practical simulations. When Gaussian probability functions were needed, we used the Box-Muller algorithm as described in Press et al. (1986).

First, the positions of the stars were randomly generated within a spherical region centered on the Sun with a radius of $4.0 \mathrm{kpc}$. For the local density of stars, a double exponential distribution is used, with a constant Galactic scale height of $250 \mathrm{pc}$ and a constant scale length of $3.5 \mathrm{kpc}$. The time at which each synthetic star was born was generated according to a constant star formation rate, and adopting a galactic disk age of $9.5 \mathrm{Gyr}$, except as otherwise stated. The mass of each star follows the standard initial mass function of Kroupa (2001). Velocities were randomly obtained taking the differential rotation of the Galaxy, the peculiar velocity of the Sun and a dispersion law which depends on the scale height into account (see García-Berro et al. 1999, for details). The evolutionary ages of the progenitors were interpolated in the BaSTI database for the appropriate metallicity (Pietrinferni et al. 2004). Given the age of the Galaxy and the age, metallicity, and mass of the progenitor star, we know which synthetic stars have had time to become white dwarfs, and for these, we derive their mass using the initial-final mass relationship of Catalán et al. (2008), except as otherwise stated.

The cooling sequences employed in this work depend on the mass of the white dwarf. If the mass of the white dwarf is less than $1.1 M_{\odot}$, a carbon-oxygen core was adopted, while we assumed that the core of the corresponding white dwarf was made of oxygen and neon if the mass was greater than this value. In our reference calculations we used the evolutionary calculations, of Renedo et al. (2010) for carbon-oxygen white dwarfs with hydrogen-rich atmospheres, and those of Benvenuto \& Althaus (1997) for hydrogen-deficient envelopes, while we used those of Althaus et al. $(2007,2005)$ for white dwarfs with oxygenneon cores. However, to assess the effects of the different cooling tracks in the white dwarf luminosity function in additional sets 
of calculations, we also employed the cooling tracks of Fontaine et al. (2011) and of the BaSTI project (Salaris et al. 2010) for carbon-oxygen white dwarfs with pure hydrogen atmospheres and those of Fontaine et al. (2011) and Bergeron et al. (2011) for white dwarfs with helium dominated atmospheres - see below. Using the appropriate white dwarf evolutionary tracks, we interpolated the luminosity, effective temperature, and the value of $\log g$ of each synthetic star. Additionally, we also interpolated their $U B V R I$ colors, which we then converted to the ugriz color system. This system is more adequate to compare our results with a sample of white dwarfs culled from the SDSS (see next section), using the transformations described in Jordi et al. (2006). To compare the sample of white dwarfs of the SSS, we used the color transformations described in $\mathrm{Xu}$ et al. (2007).

For each of the models studied below, we generated 50 independent Monte Carlo simulations (with different initial seeds), and for each of these Monte Carlo realizations, we increased the number of simulated Monte Carlo realizations to $10^{4}$ using bootstrap techniques. Using this procedure, we ensure convergence in all the final values of the relevant quantities. In what follows for each quantity of interest, we present the ensemble average of the different Monte Carlo realizations, as well as the corresponding standard deviation. Following all these steps, we were able to produce a synthetic population of disk white dwarfs, which, by definition, is complete. To this population, a series of filters, which take the observational cuts into account, must be applied. Describing them in detail is, precisely, the goal of our next section.

\section{The observational samples}

The two most recent, complete, and reliable samples of disk white dwarfs are those obtained from the SDSS and from the SSS. In this section, we describe them separately, placing special emphasis on the observational cuts employed to derive the corresponding luminosity functions. In our population synthesis study, we closely follow the prescriptions employed to obtain the observed samples with the ultimate goal of producing theoretical white dwarf populations that are as realistic as possible for both the white dwarf sample of the SDSS and for that of the SSS, since this is crucial to derive white dwarf luminosity functions that can be compared to the observational data in a meaningful way.

The SDSS surveyed $5282 \mathrm{deg}^{2}$ of high-latitude sky in five bandpasses ugriz (see Fukugita et al. 1996; Gunn et al. 1998b, 2006 for additional details) and, besides quasars and galaxies, obtained many spectra of white dwarfs and other blue stars. Using the SDSS Data Release 4, Eisenstein et al. (2006) presented a catalog of 9316 spectroscopically confirmed white dwarfs. The catalog contains both hydrogen-rich (DA) and hydrogen-deficient (non-DA) white dwarfs. From it, Harris et al. (2006) used photometric distances, USNO proper motions, and the $1 / V_{\max }$ method (Schmidt 1968) and derived a white dwarf luminosity function. In particular, to obtain this luminosity function, they considered all stars brighter than $g<19.5$. To discrimate between main sequence stars and white dwarfs they employed the reduced proper motion,

$H_{g}=g+\log \mu+5=M_{g}+5 \log V_{\tan }-3.379$,

where $\mu$ is the proper motion and $V_{\tan }$ is the tangential velocity. Specifically, they required that all objects contributing to the white dwarf luminosity function should be below and blueward the reduced proper motion corresponding to
$V_{\tan }=20 \mathrm{~km} \mathrm{~s}^{-1}$ in the reduced proper motion-color diagram. Additionally, they only selected white dwarfs with $15<g<$ 19.5 , and proper motions $\mu>0.02^{\prime \prime} \mathrm{yr}^{-1}$. The resulting sample contains about 7000 white dwarfs. Later on, De Gennaro et al. (2008), who used 3358 white dwarfs, presented an improved white dwarf luminosity function, in which only spectroscopically confirmed DA white dwarfs were employed. To obtain this luminosity function, they adopted $V_{\tan }>30 \mathrm{~km} \mathrm{~s}^{-1}$. However, given that this luminosity function does not present a cut-off, as the luminosity function of Harris et al. (2006) does, we do not use it.

The other large observational sample of disk white dwarfs is that obtained from the SSS (Rowell \& Hambly 2011). The SSS is an advanced photographic plate-digitizing machine. The plates were taken with the UK Schmidt telescope (UKST), the ESO Schmidt telescope, and the Palomar Schmidt telescope, and the resulting catalogs have been compiled by digitizing several generations of photographic Schmidt plates. The photometric system of this survey is less well known than that of the SDSS and has three passbands: $b_{\mathrm{J}}, r_{59 \mathrm{~F}}$, and $i_{\mathrm{N}}$ (see Hambly et al. 2001, for details). Using the data of several generations of these plates, Rowell \& Hambly (2011) constructed a catalog of about 10000 white dwarfs with magnitudes down to $r_{59 \mathrm{~F}} \sim 19.75$ and proper motions as low as $\mu \sim 0.05^{\prime \prime} \mathrm{yr}^{-1}$, which covers nearly three quarters of the sky. The observational selection criteria adopted to derive the corresponding white dwarf luminosity function are the following. First, the proper motion cut depends on the $b_{\mathrm{J}}$ magnitude,

$\mu>5\left(\sigma_{\mu}^{\max }\left(b_{\mathrm{J}}\right)+0.002\right)$.

In this expression, $\sigma_{\mu}$ is the standard deviation in the proper motion measurements, which means that the measured proper motion is $5 \sigma$ larger than the error at a given $b_{\mathrm{J}}$. Additionally, there is a magnitude cut $12<r_{59 \mathrm{~F}}<19.75$, whereas they also imposed a cut in the reduced proper motion diagram, by adopting $V_{\tan }>30 \mathrm{~km} \mathrm{~s}^{-1}$.

\section{Results}

Before assessing the role of the adopted metallicity law, it is convenient to check whether or not other aspects can mask its impact on the white dwarf luminosity function. For instance, the ratio of white dwarfs with hydrogen-deficient atmospheres to those with hydrogen-rich ones depends on the effective temperature, and this could possibly influence the shape of the white dwarf luminosity function. Hence, we first check if this could mask the effects of the adopted metallicity law. This is done in Sect. 4.1. The same can be said about the adopted theoretical white dwarf cooling sequences. Quite obviously, the adopted white dwarf cooling tracks also influence the precise shape of the luminosity function. Consequently, we also explore and quantify this possibility in Sect. 4.2. Finally, we analyze the effects of the adopted metallicity law in Sect. 4.3. We emphasize that all the luminosity functions presented in this section have been computed adopting a constant star formation rate.

\subsection{The fraction of non-DA white dwarfs}

To begin with, we discuss the role of the ratio of the number of white dwarfs with hydrogen-deficient atmospheres to the total number of white dwarfs, including those with hydrogen-rich atmospheres. Most white dwarf stars have hydrogen-dominated atmospheres, constituting the group of so-called DA white dwarfs. 


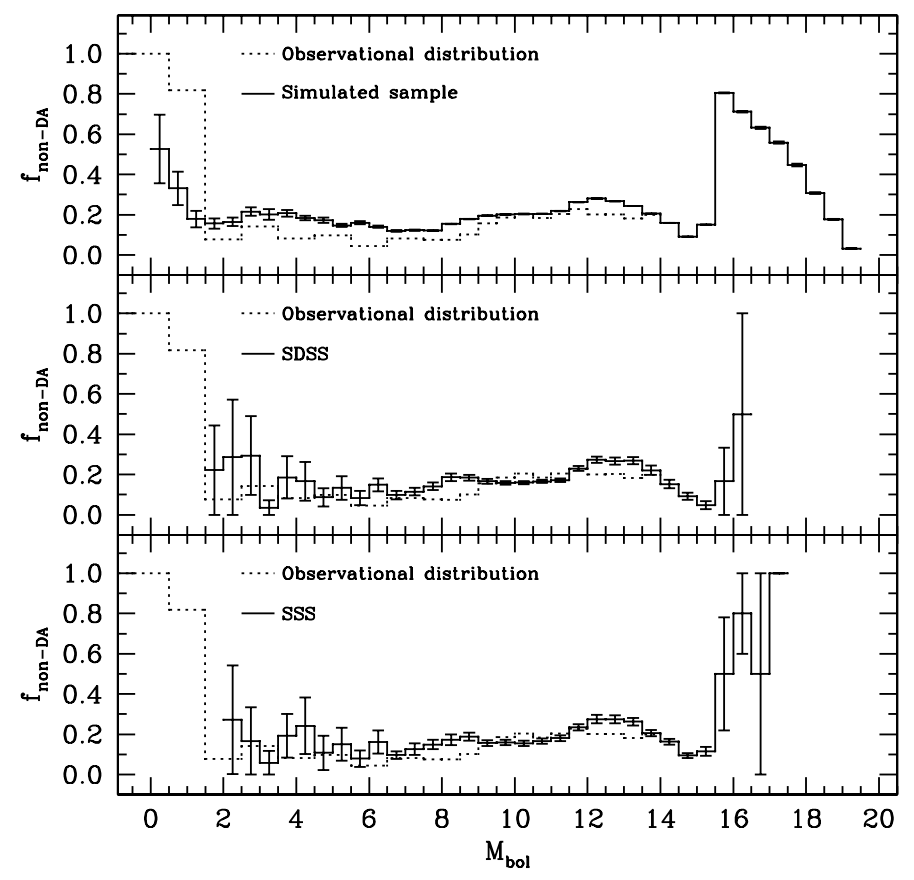

Fig. 1. Distribution of the ratio of non-DA to the total number of white dwarfs as a function of the bolometric magnitude. The solid line represents the simulated sample, whereas the dotted line corresponds to the observational data. See text for additional details.

However, hydrogen-deficient white dwarfs, known as nonDA stars, represent between $15 \%$ and $25 \%$ of the total white dwarf population of the Galaxy (Fontaine et al. 2011). In our attempt to properly analyze factors that might potentially influence the shape of the white dwarf luminosity function and given that the most recent luminosity functions incorporate both DA and non-DA white dwarfs, we first try to reproduce the observed ratio of non-DA to the total number of white dwarfs. For this set of simulations, we adopt the cooling sequences of our reference model and the evolutionary ages of white dwarf progenitors of solar metallicity. That is, we assumed that all synthetic stars have solar metallicity.

The observed ratio of non-DA white dwarfs as a function of the bolometric magnitude is shown in all three panels of Fig. 1 using a dotted line. The observational data has been compiled from Krzesinski et al. (2009) for hot white dwarfs - namely, those with effective temperatures larger than $T_{\text {eff }} \simeq 25000 \mathrm{~K}-$ and De Gennaro et al. (2008) and references therein for cooler white dwarfs. The upper panel of Fig. 1 shows the ratio of the number of non-DA white dwarfs as a function of the bolometric magnitude when all synthetic white dwarfs of an otherwise typical Monte Carlo realization are considered - that is, for a complete sample - as a solid line. This simulation was computed assuming that $20 \%$ of white dwarfs at birth have atmospheres devoid of hydrogen, while the lower two panels show the same distribution when the cuts of Harris et al. (2006) and Rowell \& Hambly (2011) are applied to the complete sample, respectively. Thus, these two last Monte Carlo realizations incorporate the biases introduced by the selection criteria. As can be seen in general, our theoretical resuls are in excellent agreement with the observational data, except at very high luminosities - namely for $M_{\text {bol }} \lesssim 2.0$ - for which the non-DA ratio predicted by our simulations for the complete sample is considerably smaller $(\sim 0.5)$ than the observed one $(\sim 1.0)$. We note, however, that the number density of white dwarfs is very small

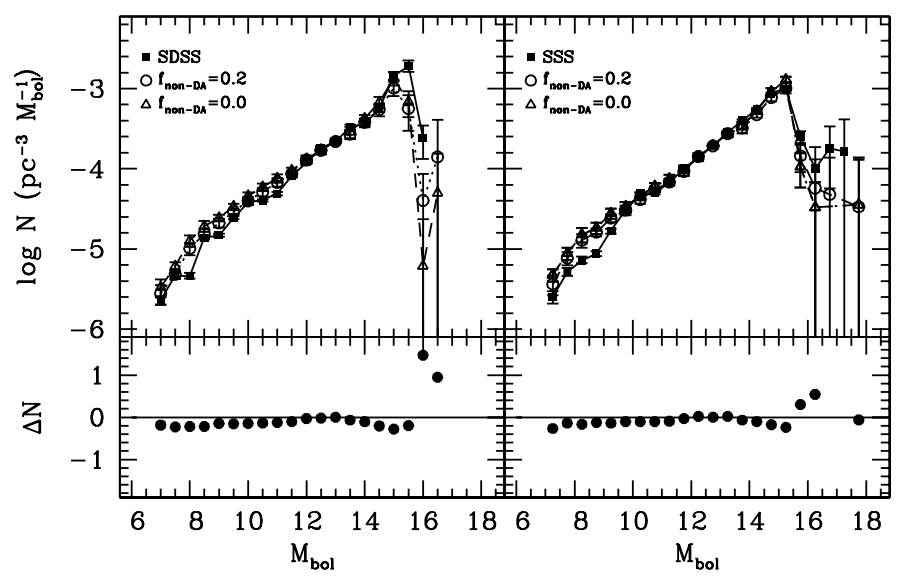

Fig. 2. Simulated white dwarf luminosity function for a pure DA white dwarf population (open triangles) and for a population in which a canonical fraction of $20 \%$ of non-DA white dwarfs (open circles) is adopted. Both luminosity functions are compared with the observational white dwarf luminosity function (solid squares) of Harris et al. (2006; left top panel) and Rowell \& Hambly (2011; right top panel). In the bottom panels, we show the residuals between the synthetic luminosity functions.

at these bolometric magnitudes and, hence, this ratio presents large fluctuations, depending on the specific Monte Carlo realization chosen. It is worth noting that the effects of the selection criteria for this range of bolometric magnitudes is important as well, as the ratio of non-DA white dwarfs turns out to be $\sim 0.5$ for the complete sample. This ratio drops to $\sim 0.0$ for the two restricted samples, although the statistical fluctuations are very large. Another interesting aspect of the theoretical results that is also worth commenting is that at very low luminosities, our population synthesis calculations predict a noticeable increase of the ratio of non-DA to DA white dwarfs for the complete sample, which agrees with what is found for the local sample of white dwarfs (see Giammichele et al. 2012, and references therein). This is evident as well for the sample of white dwarfs in which the selection criteria of Rowell \& Hambly (2011) are used. If the selection criteria of Harris et al. (2006) are employed, the ratio of non-DA white dwarfs does not show such a marked increase because very few low luminosity white dwarfs are found when this set of criteria is adopted. We elaborate on this below when discussing the white dwarf luminosity function.

Once established that the adopted $20 \%$ ratio of non-DA white dwarfs at birth results in a credible distribution for all luminosities, we test the actual impact of this ratio on the white dwarf luminosity function. To this end we compute an additional set of Monte Carlo simulations for which we adopt $f_{\text {non-DA }}=0.0$ and we compare the corresponding luminosity function with that obtained using $f_{\text {non-DA }}=0.2$. The result of this exercise is shown in Fig. 2. In the top panels of this figure the luminosity functions obtained when $f_{\text {non-DA }}=0.0$ and $f_{\text {non-DA }}=0.2$ are compared, while the observed luminosity functions of Harris et al. (2006) and Rowell \& Hambly (2011) are also displayed in the respective panels. Obviously, to produce these luminosity functions, the corresponding selection criteria have been used. The bottom panels of this figure display the residuals between both theoretical luminosity functions, which help in assessing the differences between both simulations, as defined as

$\Delta N=2 \frac{N_{f_{\mathrm{DB}}=0.2}-N_{f_{\mathrm{DB}}=0}}{N_{f_{\mathrm{DB}}=0.2}+N_{f_{\mathrm{DB}}=0}}$. 
As can be seen in the bottom panels of Fig. 2, the effect of the adopted non-DA ratio on the simulated white dwarf luminosity functions is minimal, except for bolometric magnitudes larger than $M_{\text {bol }} \simeq 15$. For the bins with bolometric magnitudes larger than this value, the differences are noticeable in both cases, and the luminosity function in which $f_{\text {non-DA }}=0.2$ is in better agreement with the observational one. In particular, when $f_{\text {non-DA }}=$ 0.2 , is adopted the number density of low-luminosity white dwarfs increases, as should be expected, given that white dwarfs with hydrogen-defficient atmospheres cool faster. Actually, the number density of white dwarfs for the luminosity functions computed with $f_{\text {non-DA }}=0.0$ is consistent with zero, since for these bins we obtain at most only one object per bin in all Monte Carlo realizations. We thus conclude that the hot branch of the white dwarf luminosity function is almost insensitive to the adopted ratio of non-DA white dwarfs and that the effects of this ratio concentrate in the poorly determined bins with the lowest luminosities.

\subsection{The effects of the adopted cooling tracks}

We now discuss which set of cooling sequences best reproduces the shape of the observed white dwarf luminosity function. To this end in Fig. 3, we compare various sets of evolutionary cooling sequences with the two most recent and reliable observational white dwarf luminosity functions, namely that of Harris et al. (2006) and that of Rowell \& Hambly (2011). The data shown in this figure corresponds to the ensemble average of several single independent Monte Carlo realizations, as described before, with the inputs described in Sect. 2. In the top panels of this figure, we display the results of such comparison when the luminosity functions obtained using the cooling tracks of Fontaine et al. (2011) are compared to those obtained using our reference model, as done in Sect. 2, whereas a comparison of the results obtained when the cooling sequences of Salaris et al. (2010) and those obtained when the cooling tracks of Renedo et al. (2010) are used is performed in the bottom panels. In each panel, we also show the residuals between both theoretical simulations. As we did previously for all the calculations shown in this figure, we adopted a constant metallicity equal to the solar value.

Figure 3 demonstrates that all three sets of cooling sequences match the bright branch of both observational white dwarf luminosity functions very well and that there are not appreciable differences between all three luminosity functions at high luminosities. This agreement is even better when the white dwarf luminosity function of Rowell \& Hambly (2011) is adopted, as the location of the maximum of the white dwarf luminosity function is nicely fit by all three sets of cooling tracks. Now, we turn our attention to the cool branch of the white dwarf luminosity function and the shape of its drop-off. Again, all three sets of cooling sequences are in excellent agreement with the observational data, although we emphasize that selection effects may play a key role at these very low luminosities and both the Monte Carlo simulated sample and the real ones suffer from poor statistics. Nevertheless, we note that all three sets of calculations reproduce the downturn at very faint magnitudes of the luminosity function obtained from the SSS, which is not present in the luminosity function of Harris et al. (2006) because of the sample selection procedures. Given these results, there are no objective reasons for discarding any set of evolutionary sequences, and hence for the rest of the paper, we adopt the cooling sequences of our fiducial model, which is based on the theoretical cooling sequences of Renedo et al. (2010).
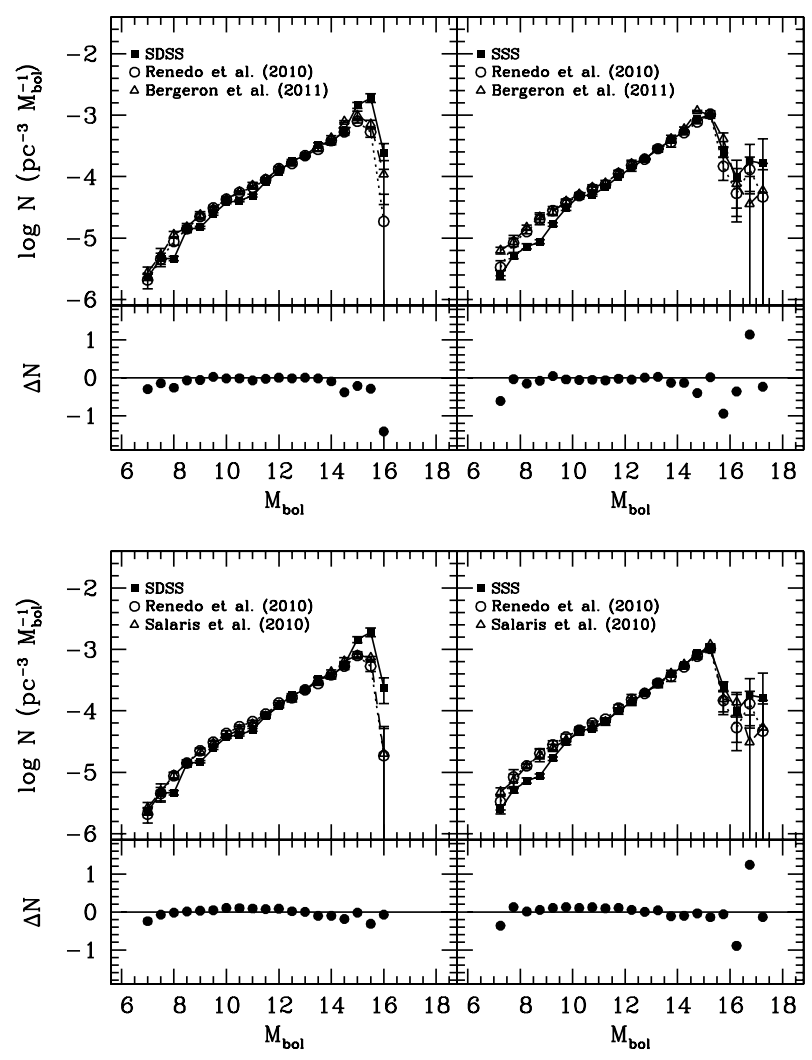

Fig. 3. Simulated white dwarf luminosity functions computed using our reference model (open circles) compared to those obtained using the cooling sequences of Fontaine et al. (2011; top panels, open triangles) and Salaris et al. (2010; bottom panels, also open triangles). Also shown are the observational white dwarf luminosity function (solid squares) of Harris et al. (2006; left panels) and Rowell \& Hambly (2011; right panels). The synthetic white dwarf luminosity functions have been normalized to the observational point with minimum error bars.

\subsection{The influence of the metallicity law}

Metallicity is known to have a considerable influence in age estimations obtained when the turn-off of main sequence stars of stellar clusters is employed to derive the corresponding age. Hence, it is natural to wonder whether or not the age derived from the drop-off of the disk white dwarf luminosity function depends on the adopted age-metallicity relationship. To perform this analysis, we adopted two different metallicity laws. The first one is the classical metallicity law of Twarog (1980), which predicts that the metallicity of white dwarf progenitor stars monotonically increases from zero at very early ages of our Galaxy to solar metallicity for the current age of the Galactic disk. Specifically, the metallicities of the individual synthetic stars are randomly drawn by following a Gaussian distribution centered in $F(t)$, where $F(t)$ is a polynomial fit of the results presented in Fig. 1 of Bravo et al. (1993) with a dispersion $\sigma=0.1$. Our second age-metallicity relationship is based on the observational data compiled by the Geneva-Conpenhague survey (Casagrande et al. 2011). This recent study predicts that the metallicity of the Galactic disk is constant with time but has a sizable dispersion. We note that this distribution of metallicities has been confirmed by the recent results of Duran et al. (2013). Thus, for the same age, white dwarf progenitors span a broad range of metallicities. In particular, for each synthetic star, we employ $[\mathrm{Fe} / \mathrm{H}]=[\mathrm{Fe} / \mathrm{H}]_{\odot}+\Delta[\mathrm{Fe} / \mathrm{H}]$, where $\Delta[\mathrm{Fe} / \mathrm{H}]$ is randomly drawn 

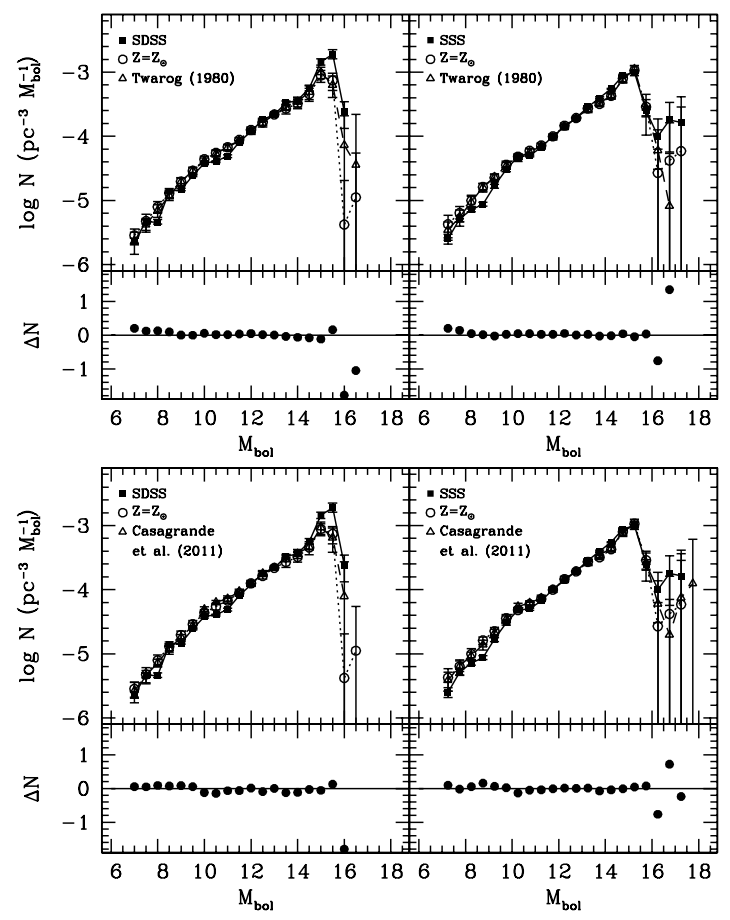

Fig. 4. Simulated white dwarf luminosity function using the metallicity law of Twarog (1980; top panels) and that of Casagrande et al. (2011; bottom panels) compared to the observational white dwarf luminosity functions of Harris et al. (2006; left panels) and Rowell \& Hambly (2011; right panels). As in previous figures, we also show the residuals between the synthetic luminosity functions computed with a given metallicity law, and the luminosity function computed adopting the solar value (see text for details).

from a Gaussian distribution with the metallicity spread found by Casagrande et al. (2011), $\sigma=0.4$.

Whatever the adopted age-metallicity relationship is in both cases we interpolate both the main-sequence lifetimes of the progenitors and the final masses, cooling times, colors and luminosities of the resulting white dwarfs. For this analysis, we adopt the set of cooling sequences of Renedo et al. (2010), which almost cover the full range of metallicities. If a progenitor star has metallicity lower than $Z=10^{-3}$, the lowest metallicity of the evolutionary sequences of Renedo et al. (2010), we adopt the values predicted by these calculations. If the metallicity of the progenitor stars is larger than $Z=10^{-2}$, we interpolate between the evolutionary sequences of Renedo et al. (2010) and those of Althaus et al. (2010b), which have a maximum metallicity of $Z=0.06$, which is enough for our purposes. Finally, we adopt an age for the Galactic disk of 9.5 Gyr.

Our results are presented in Fig. 4. In particular, we compare the luminosity functions obtained using the age-metallicity relationship of Twarog (1980) and using a fixed value of the metallicity equal to the solar one with the observational luminosity functions of the SDSS and the SSS in the upper panels of this figure. In the bottom panels, we show the same comparison, but this time using the metallicity law of Casagrande et al. (2011). As in previous figures, we also show the corresponding differences between the theoretical calculations. As can be seen in this figure, both theoretical calculations yield almost the same results, and most importantly, the position of the theoretical cut-off of the white dwarf luminosity function is not affected by the adopted metallicity law, no matter if the adopted metallicity law is that of Twarog (1980), that of Casagrande et al. (2011), or if a fixed value of the metallicity is adopted. This, in turn, means that the age estimate of the solar neighborhood obtained from the location of the drop-off of the white dwarf luminosity function is robust. Actually, the age difference between the theoretical calculations is rather small. For instance, when the luminosity function of the SDSS is adopted, the age of the disk derived using the metallicity law of Casagrande et al. (2011) is obtained to be 9.5 Gyr. If the metallicity law of Twarog (1980) is used, however, the age turns out to be $10 \mathrm{Gyr}$, and if a fixed value of the metallicity equal to the solar one is adopted, we obtain $9.8 \mathrm{Gyr}$. These ages when the adopted luminosity function is that of the SSS are 10 Gyr for the first two cases and 10.3 Gyr for the case in which a constant solar metallicity with no dispersion is employed.

At first look, this result may seem surprising since at the luminosity of the cut-off contribute the oldest white dwarfs in the Galaxy. These white dwarfs have cooling ages, which are approximately equal to the age of the Galactic disk, and thus have negligible main-sequence lifetimes, which translates in small metallicities for the age-metallicity relationship of Twarog (1980). However, since these white dwarfs have progenitors with very short main sequence lifetimes, it also means that their progenitors are rather massive. Consequently, the steep slope of the initial mass function prevents the formation of a large number of these stars. On the contrary, the peak of the white dwarf luminosity function is dominated by white dwarfs of $\sim 0.6 \pm 0.05 M_{\odot}$, which are the bulk of white dwarfs in our solar neighborhood, and which have very different main sequence lifetimes (and metallicities). The net result is that the drop-off of the white dwarf luminosity function is very abrupt, and its position is mainly determined by otherwise typical white dwarfs. Additionally, for the case of the metallicity law of Casagrande et al. (2011), the relatively large dispersion of metallicities contributes to erase the metallicity dependence of stellar lifetimes and results in a negligible dependence of the position of the dropoff of the white dwarf luminosity function on the metallicity of white dwarf progenitors.

Given that the position of the cut-off of the theoretical white dwarf luminosity function does not depend on the adopted metallicity law, we computed the age of the disk that best fits the observed distribution of white dwarfs. The resulting white dwarf luminosity functions are displayed in Fig. 5, and the corresponding ages are $9.5 \mathrm{Gyr}$ for the case in which the white dwarf luminosity function of the SDSS is employed, while we obtain 10.0 Gyr when the luminosity function of the SSS is adopted. Thus, currently, the age determination uncertainty of the Galactic disk using the cut-off of the white dwarf luminosity function seems to be dominated by the relatively small number of white dwarfs in the lowest luminosity bins. A comparison of the SDSS and SSS luminosity functions indicates that this introduces an uncertainty of $\sim 0.5 \mathrm{Gyr}$.

\section{Summary and conclusions}

In this paper, we explored the possible dependence of the position of the white dwarf luminosity function on the adopted agemetallicity relationship. Our motivation for undertaking such a study was that most age estimators depend on the metallicity of the adopted theoretical stellar evolutionary models, and such a study was lacking for the case in which the age of the Galactic disk is estimated using the position of the cut-off of the white dwarf luminosity function. Our study fills this gap. In doing so, we used an up-to-date Monte Carlo simulator, which incorporates the most recent and reliable prescriptions for both the 
R. Cojocaru et al.: The effects of the metallicity on the population of disk white dwafs

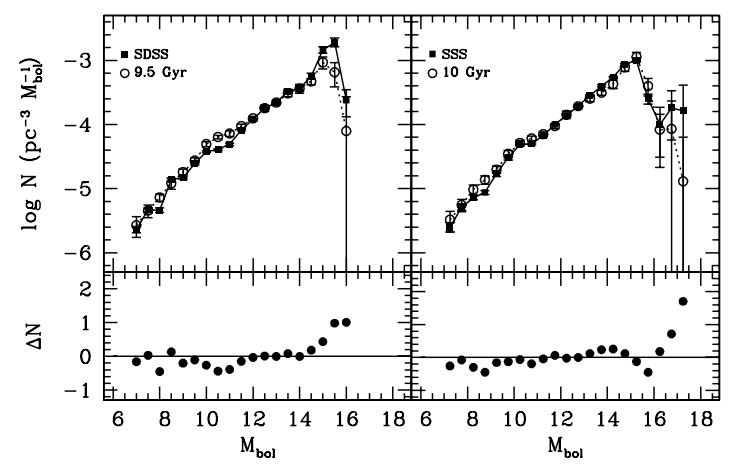

Fig. 5. Simulated white dwarf luminosity functions using the very recent metallicity law of Casagrande et al. (2011), compared to the observed luminosity functions of the SDSS (left panel) and the SSS (right panel). The adopted disk ages are 9.5 and $10.0 \mathrm{Gyr}$, respectively, which are the ages that best reproduce the position of the observed cut-off of the luminosity function. As done in previous figures, we also show the corresponding residuals.

main sequence lifetimes and cooling ages, as well as the most realistic Galactic inputs. We also adopted two typical and frequently used metallicity laws, the first one being the classical age-metallicity relationship of Twarog (1980), which displays a monotonically increasing trend, and the metallicity law of Casagrande et al. (2011), which does not increase as time passes by, but instead shows a relatively large dispersion around the solar value. Nevertheless, before studying the role of the adopted age-metallicity relationship, we also studied other possible effects that may eventually have a noticeable impact on the shape of the white dwarf luminosity function. Specifically, we studied how the ratio of non-DA to DA white dwarfs influences the shape of the luminosity function and of its cut-off and also how the choice of the adopted theoretical degenerate cooling sequences affects the luminosity function. We found that neither the shape of the bright portion of the white dwarf luminosity function nor the position of its downturn at low luminosities are noticeably affected by these inputs. More importantly, we also found that the adopted age-metallicity relationship has a negligible impact on the shape of the luminosity function and on the position of its drop-off. Hence, the age estimates of the Galactic disk obtained from the number counts of low-luminosity white dwafs are robust, and the age differences mainly come from the way in which low-luminosity white dwarfs are culled to obtain a nearly complete sample. For the two most recent observational determinations of the white dwarf luminosity function - namely, that of Harris et al. (2006), which was obtained from white dwarfs found in the SDSS - and that of Rowell \& Hambly (2011), which was derived using data from the SSS - we obtain 9.5 Gyr and 10.0 Gyr, respectively.

Acknowledgements. This research was supported by AGAUR, by MCINN grant AYA2011-23102, and by the European Union FEDER funds. R.C. also acknowledges financial support from the FPI grant with reference BES-2012053448 .

\section{References}

Althaus, L. G., García-Berro, E., Isern, J., \& Córsico, A. H. 2005, A\&A, 441, 689

Althaus, L. G., García-Berro, E., Isern, J., Córsico, A. H., \& Rohrmann, R. D. 2007, A\&A, 465, 249

Althaus, L. G., Córsico, A. H., Isern, J., \& García-Berro, E. 2010a, A\&ARv, 18, 471

Althaus, L. G., García-Berro, E., Renedo, I., et al. 2010b, ApJ, 719, 612

Benvenuto, O. G., \& Althaus, L. G. 1997, MNRAS, 288, 1004
Bergeron, P., Wesemael, F., Dufour, P., et al. 2011, ApJ, 737, 28

Bravo, E., Isern, J., \& Canal, R. 1993, A\&A, 270, 288

Casagrande, L., Schönrich, R., Asplund, M., et al. 2011, A\&A, 530, A138

Catalán, S., Isern, J., García-Berro, E., \& Ribas, I. 2008, MNRAS, 387, 1693

De Gennaro, S., von Hippel, T., Winget, D. E., et al. 2008, AJ, 135, 1

Diaz-Pinto, A., Garcia-Berro, E., Hernanz, M., Isern, J., \& Mochkovitch, R. 1994, A\&A, 282, 86

Dreiner, H. K., Fortin, J.-F., Isern, J., \& Ubaldi, L. 2013, Phys. Rev. D, 88, 043517

Duran, Ş., Ak, S., Bilir, S., et al. 2013, PASA, 30, 43

Eisenstein, D. J., Liebert, J., Harris, H. C., et al. 2006, ApJS, 167, 40

Fontaine, G., Brassard, P., \& Bergeron, P. 2011, PASP, 113, 409

Fukugita, M., Ichikawa, T., Gunn, J. E., et al. 1996, AJ, 111, 1748

García-Berro, E., Hernanz, M., Isern, J., \& Mochkovitch, R. 1988, Nature, 333, 642

Garcia-Berro, E., Hernanz, M., Isern, J., \& Mochkovitch, R. 1995, MNRAS, 277,801

García-Berro, E., Torres, S., Isern, J., \& Burkert, A. 1999, MNRAS, 302, 173

García-Berro, E., Torres, S., Isern, J., \& Burkert, A. 2004, A\&A, 418, 53

García-Berro, E., Torres, S., Althaus, L. G., et al. 2010, Nature, 465, 194

García-Berro, E., Lorén-Aguilar, P., Torres, S., Althaus, L. G., \& Isern, J. 2011, J. Cosmol. Astropart. Phys., 5, 21

Geijo, E. M., Torres, S., Isern, J., \& García-Berro, E. 2006, MNRAS, 369, 1654

Giammichele, N., Bergeron, P., \& Dufour, P. 2012, ApJS, 199, 29

Gunn, J. E., Carr, M., Rockosi, C., et al. 1998a, AJ, 116, 3040

Gunn, J. E., Carr, M., Rockosi, C., et al. 1998b, AJ, 116, 3040

Gunn, J. E., Siegmund, W. A., Mannery, E. J., et al. 2006, AJ, 131, 2332

Hambly, N. C., MacGillivray, H. T., Read, M. A., et al. 2001, MNRAS, 326, 1279

Hansen, B. M. S., Brewer, J., Fahlman, G. G., et al. 2002, ApJ, 574, L155

Hansen, B. M. S., Kalirai, J. S., Anderson, J., et al. 2013, Nature, 500, 51

Harris, H. C., Munn, J. A., Kilic, M., et al. 2006, AJ, 131, 571

Haywood, M. 2008, MNRAS, 388, 1175

Isern, J., Hernanz, M., Mochkovitch, R., \& Garcia-Berro, E. 1991, A\&A, 241, L29

Isern, J., Mochkovitch, R., Garcia-Berro, E., \& Hernanz, M. 1997, ApJ, 485, 308

Isern, J., García-Berro, E., Hernanz, M., Mochkovitch, R., \& Torres, S. 1998, ApJ, 503, 239

Isern, J., García-Berro, E., Hernanz, M., \& Chabrier, G. 2000, ApJ, 528, 397

Isern, J., García-Berro, E., Domíguez, I., Salaris, M., \& Straniero, O. 2005, in 14th European Workshop on White Dwarfs, eds. D. Koester, \& S. Moehler, ASP Conf. Ser., 334, 43

Isern, J., García-Berro, E., Torres, S., \& Catalán, S. 2008, ApJ, 682, L109

James, F. 1990, Comput. Phys. Commun., 60, 329

Jeffery, E. J., von Hippel, T., DeGennaro, S., et al. 2011, ApJ, 730, 35

Jordi, K., Grebel, E. K., \& Ammon, K. 2006, A\&A, 460, 339

Kalirai, J. S., Ventura, P., Richer, H. B., et al. 2001, AJ, 122, 3239

Kawaler, S. D. 1996, ApJ, 467, L61

Kroupa, P. 2001, MNRAS, 322, 231

Krzesinski, J., Kleinman, S. J., Nitta, A., et al. 2009, å, 508, 471

Mochkovitch, R., Garcia-Berro, E., Hernanz, M., Isern, J., \& Panis, J. F. 1990, A\&A, 233, 456

Noh, H.-R., \& Scalo, J. 1990, ApJ, 352, 605

Nordström, B., Mayor, M., Andersen, J., et al. 2004, A\&A, 418, 989

Olsen, E. H. 1983, A\&AS, 54, 55

Pauli, E.-M., Napiwotzki, R., Altmann, M., et al. 2003, A\&A, 400, 877

Pietrinferni, A., Cassisi, S., Salaris, M., \& Castelli, F. 2004, ApJ, 612, 168

Press, W. H., Flannery, B. P., \& Teukolsky, S. A. 1986, Numerical Recipes. The art of scientific computing (Cambridge: University Press)

Renedo, I., Althaus, L. G., Miller Bertolami, M. M., et al. 2010, ApJ, 717, 183

Rohrmann, R. D., Althaus, L. G., García-Berro, E., Córsico, A. H., \& Miller Bertolami, M. M. 2012, A\&A, 546, A119

Rowell, N. 2013, MNRAS, 434, 1549

Rowell, N., \& Hambly, N. C. 2011, MNRAS, 417, 93

Salaris, M., Cassisi, S., Pietrinferni, A., Kowalski, P. M., \& Isern, J. 2010, ApJ, 716,1241

Salaris, M., Althaus, L. G., \& García-Berro, E. 2013, A\&A, 555, A96

Schmidt, M. 1968, ApJ, 151, 393

Segretain, L., Chabrier, G., Hernanz, M., et al. 1994, ApJ, 434, 641

Sellwood, J. A., \& Binney, J. J. 2002, MNRAS, 336, 785

Strömgren, B. 1987, in NATO ASIC Proc. 207: The Galaxy, eds. G. Gilmore, \& B. Carswell, 229

Torres, S., García-Berro, E., Burkert, A., \& Isern, J. 2002, MNRAS, 336, 971

Torres, S., García-Berro, E., \& Isern, J. 2007, MNRAS, 378, 1461

Twarog, B. A. 1980, ApJ, 242, 242

Winget, D. E., Hansen, C. J., Liebert, J., et al. 1987, ApJ, 315, L77

Xu, Y., Deng, L. C., \& Hu, J. Y. 2007, MNRAS, 379, 1373 